\title{
Creation and control of new morphologies via supramacromolecular self-assembly
}

\author{
Yushu Matsushita, Atsushi Noro and Atsushi Takano
}

\begin{abstract}
Various supramacromolecular self-assembly mechanisms of complex polymer systems via non-covalent bonding interactions were summarized in this article. Basically, three types of systems were introduced. The first class is the morphological transition of block copolymer/homopolymer blends with hydrogen bonds (HBs) or block copolymer/metal salt hybrids via complex formation. Systematic morphological transition was observed upon the addition of a homopolymer or metal salt, depending on its composition of volume fraction. The second class consists of the formation of block copolymer-type supramacromolecules via hydrogen bonding between two chemical moieties connected at one end of each polymer chain, resulting in new nanophase-separated structures. As an advanced branch of this second class, new morphologies were observed in block copolymer/block copolymer blends with HBs. The third class is supramacromolecular gelation, which was observed for ABA triblock copolymer/C homopolymer or telechelic polymer/homopolymer blends, both including complementary electron donors/acceptors.
\end{abstract}

Polymer Journal (2012) 44, 72-82; doi:10.1038/pj.2011.81; published online 21 September 2011

Keywords: block copolymer; hydrogen bonding; nanophase-separated structure; self-assembly; supramacromolecule; supramolecular polymer gel

\section{INTRODUCTION}

The idea of 'supramolecule' is relatively new, considering the long history of modern chemistry. Researchers in this field admit that the basic idea, which was summarized in 1980s by Lehn et al. ${ }^{1}$ opened the door to new chemistry and physics because the supramolecular assembly of molecules or chemical compounds produces new classes of functional materials through simpler processes than the traditionally used covalent bond construction.

The introduction of this idea into polymer chemistry or physics is even newer, occurring in the late 1980s. If we classify non-covalent bonding interactions very simply, they basically belong to three categories: (1) ionic bonding interactions, including acid-base type ones; (2) hydrogen bonds (HBs), including complementary bio-based pairs; and (3) metal-to-ligand coordination that functions via complex formation between metal compounds and organic molecules as electron acceptors.

As a pioneering work on the first category, Russell $e t a l^{2}$ reported on the block-copolymer-type supramolecular self-assembly from mixtures of two telechelic polymers: one end-capped with a tertiary amine and the other terminated with sulfonate or carboxylate moieties. Later, Haraguchi et al. ${ }^{3,4}$ studied the aggregate formation of $\mathrm{NH}_{2}$-terminated polystyrene (S) and $\mathrm{SO}_{3} \mathrm{H}$-terminated polyethyleneglycol in toluene; in addition, the thermal tunability of lamellar spacing in block copolymer-type supramolecules from $\mathrm{NH}_{2}$-terminated polyisoprene (I) and telechelic $\mathrm{SO}_{3} \mathrm{H}$-terminated $\mathrm{S}$ in bulk has been investigated. ${ }^{5}$ Moreover, Pispas et al. ${ }^{6}$ observed the formation of a miktoarm block copolymer of the type $\mathrm{SI}_{3}$, where $\mathrm{S}$ denotes polystyrene with a terminal trifunctional group of dimethyl amine and I is polyisoprene terminated with $\mathrm{SO}_{3} \mathrm{H}$. More recently, Noro et al. ${ }^{7}$ studied the stoichiometric effects on nanophase-separated structures of block- and graft-type supramacromolecules using a pair of $\mathrm{SO}_{3} \mathrm{H}$ and $\mathrm{NH}_{2}$ moieties, whereas Qian et al. ${ }^{8}$ reported on the preparation of supramolecular graft copolymers from poly (4-vinylpyridine) and poly( $N$-vinylpyrrolidone) terminated with a $\mathrm{COOH}$ group.

There are a variety of studies on self-assembly via hydrogen bonding. An earlier attempt using multiple HBs was reported by Sijbesma et al., ${ }^{9}$ who used a 2-ureido-4-pyrimidone terminated long alkyl chain, which produces dimers reversibly with or without quadruple HBs; furthermore, supramolecular polymer formation by multiple hydrogen bonding arrays was proposed to be feasible. ${ }^{10}$ Moreover, the formation of pseudo-block copolymers with multiple hydrogenbonding moieties has been examined, ${ }^{11-14}$ and the thermoreversible structure formation of poly(alkyl acrylates) with multiple hydrogen bonding moieties was observed. ${ }^{15,16}$

Complex supramacromolecular self-assembly with the use of $\mathrm{HB}$ formation between poly(vinylpyridine) and phenol groups has been studied by many research groups. ${ }^{17-20}$ Dai et al. ${ }^{21}$ reported on interpolymer complex formation between poly(4-hydroxystyrene) (H) and poly(vinylpyridine) (P). This $\mathrm{H} / \mathrm{P}$ combination was extensively 
adopted later. The self-assembly of two diblock copolymers containing $\mathrm{H}$ and $\mathrm{P}$ block sequences have been examined; ${ }^{22}$ in addition, an even more complex system was examined, and hierarchical structures have been found in triblock/diblock copolymer blend systems. ${ }^{23}$ Several extensive studies on diblock copolymer/diblock copolymer and triblock copolymer/diblock copolymer blends with HBs will be introduced in detail later in this article.

Metallo-supramolecular polymers, ${ }^{24,25}$ with the aid of metal/organic compound complex formation, can be used to produce high-performance and promising materials. The incorporation of metal nanoclusters, such as silver, gold and palladium, into microphase-separated phosphine-containing block copolymers with various morphologies was first examined by Cohen et al. ${ }^{26}$ The periodic distribution of gold nanoparticles with HBs between diamino triazine and thymine were studied. ${ }^{27}$ The phase transformation from a cylinder to a lamellar structure with the introduction of gold was examined, ${ }^{28}$ and the association strength of gold, silver and copper ions with pyridine groups in poly(4-vinylpyridine-b-e-caprolactone) was determined in detail by the same research group. ${ }^{29,30}$ Selective incorporation of metal nanoparticles into polyvinylpyridine chains in block copolymers was investigated for palladium, ${ }^{31,32}$ cadmium $^{33,34}$ and gold. ${ }^{35,36}$

In this article, the recent advances in supramacromolecular selfassembly of block copolymers or related complex systems that were made by our research groups are summarized. The scientific achievements can be divided into three categories. The first category is the morphological transition of block copolymer/homopolymer blends with hydrogen bonding or block copolymer/metal salt hybrids via complex formation. The second category consists of forming block copolymer-type supramacromolecules via hydrogen bonding between two chemical moieties of each main polymer end. The third category is supramacromolecular gelation, which was observed for ABA triblock copolymer/C homopolymer or telechelic polymer/homopolymer blends, both of which include complementary electron donors/ acceptors.

\section{SUPRAMACROMOLECULAR SELF-ASSEMBLY}

The morphological transition of block copolymers with additives produces non-covalent bonding interactions

The morphologies of block copolymers can be controlled mainly by the composition of the component-polymers. If a 'composition change' is required for morphology transition, this normally involves syntheses of many block copolymers with different compositions. Another easier method of changing the composition is to add component homopolymers to parent block copolymers because a low-molecular-weight homopolymer can dissolve in the domains of block chains and affect the interfacial structure of the system, which induces morphological transition. However, the effects of this procedure on morphology change are quite limited because added homopolymer chains tend to be expelled spontaneously from the domains of the block chains when the amount of homopolymer chains exceeds the solubility limit, which is not extremely high, even though the molecular weight of an added homopolymer is fairly low.

The utilization of hydrogen bonding might be a novel method to overcome this drawback, if the proton donor/acceptor stoichiometry is not a sensitive controlling factor. Among many donor/acceptor pairs, we selected the combination of poly(4-hydroxystyrene) $(\mathrm{H})$ as a donor and poly(vinylpyridine) (P) as an acceptor. Then, the morphology change of block copolymer/homopolymer blends was investigated precisely using poly(styrene-b-2-vinylpyridine) as a block copolymer and $\mathrm{H}$ as a homopolymer. ${ }^{37,38}$ Block copolymers for this study were prepared by anionic polymerizations in tetrahydrofuran, ${ }^{39,40}$ whereas an $\mathrm{H}$ homopolymer was also synthesized by an anionic polymerization of poly(4-tert-butoxystyrene) followed by a hydrolysis reaction. ${ }^{41-44}$ Sample films were obtained by casting a solvent from the solution of tetrahydrofuran. Four images at the bottom of Figure 1 show the morphology change of the polystyrene-b-poly(2-vinylpyridine) (SP-73), whose molecular weight is $130 \mathrm{kDa}$ and the volume fraction of the polystyrene (S) block is 0.67 , upon the addition of $\mathrm{H}$ with a molecular weight of $14 \mathrm{kDa}$. These images are compared with

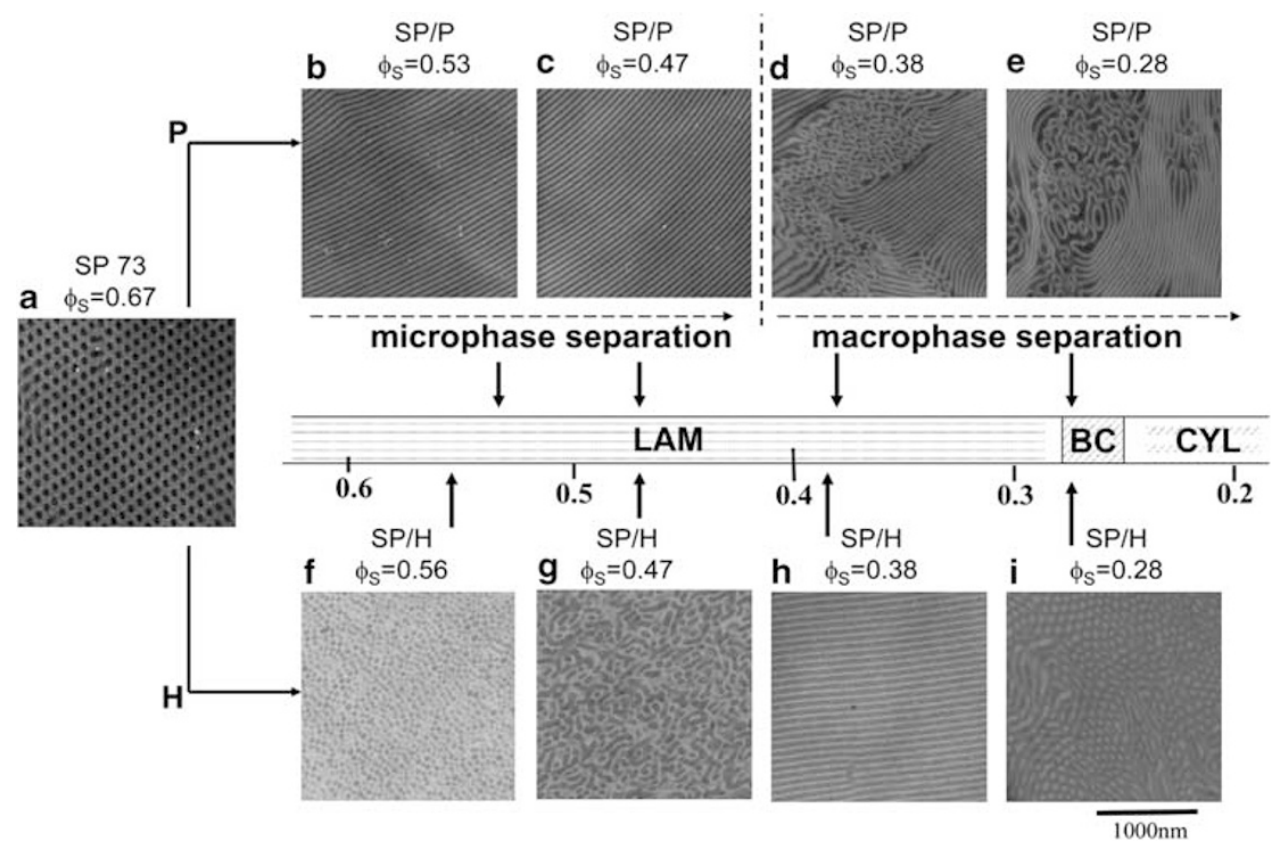

Figure 1 Transmission electron microscopy (TEM) micrographs showing different structures arising from mixing a block copolymer, poly(styrene-b-2vinylpyridine) 73 (a), with the homopolymers $\mathrm{P}$ (b-e) and $\mathrm{H}(\mathbf{f}-\mathbf{i})$. Reprinted with permission from Dobrosielska et al. ${ }^{37}$ Copyright (C) 2008 . American Chemical Society. 

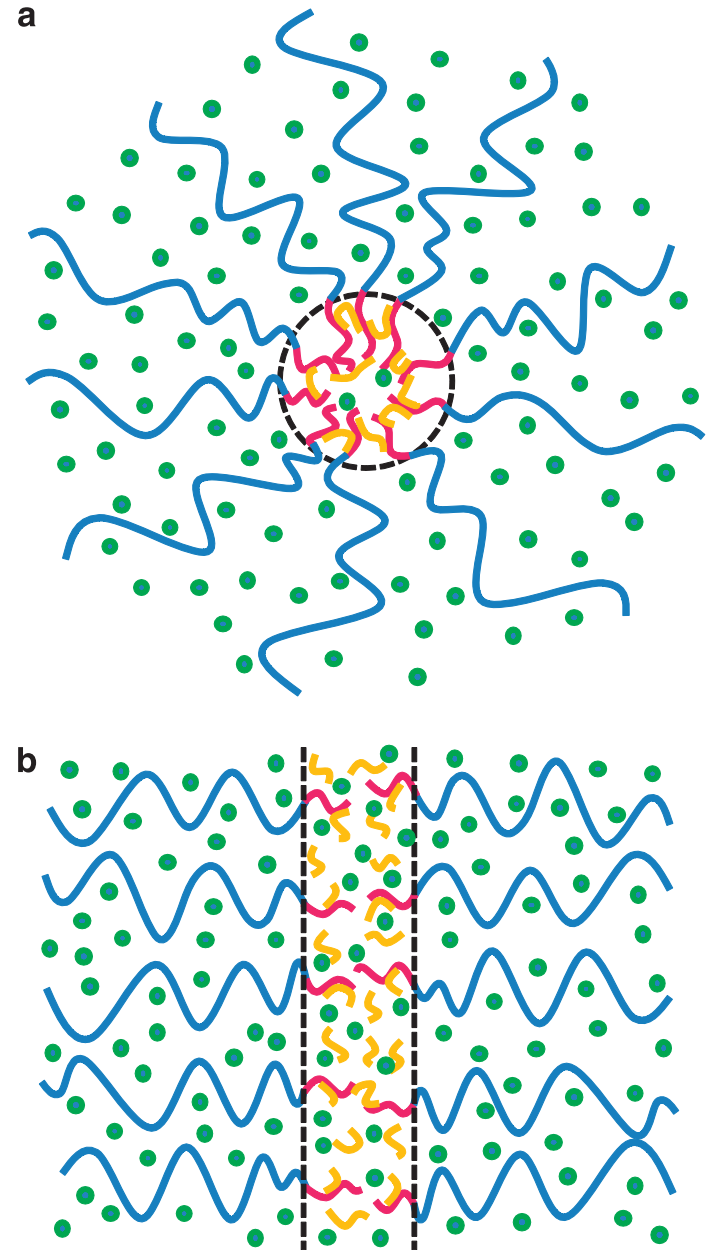

Figure 2 The formation of spherical domains owing to the production of aggregates between $\mathrm{P}$ block chains and $\mathrm{H}$ homopolymer chains (a), and a more periodic lamellar domain structure due to the stabilizing effect of added $\mathrm{H}$ homopolymer in the $\mathrm{P}+\mathrm{H}$ mixed phase (b).

the other four images at the top of Figure 1, showing the structural change of the copolymer upon the addition of a P2VP homopolymer $(6.6 \mathrm{kDa})$. First, it is interesting that a cylindrical structure of the parent polymer transitions to a spherical structure when the $\mathrm{S} /(\mathrm{P}+\mathrm{H})$ ratio is $0.56 / 0.44$, as shown in Figure 1f. This reverse morphological change, regardless of the addition of an $\mathrm{H}$ homopolymer, is due to the film preparation process, in which a $\mathrm{P}+\mathrm{H}$ complex starts to form aggregates upon casting a solvent, resulting in the formation of spherical domains, which remains until the final stage of domain formation. However, this abnormal morphology disappears if the amount of the added $\mathrm{H}$ increases up to an $\mathrm{S} /(\mathrm{P}+\mathrm{H})$ ratio of $0.47 /$ 0.53 , as is shown in Figure $1 \mathrm{~g}$. This phenomenon probably indicates that the excess amount of $\mathrm{H}$ chains can have the role of a shield-like compound, preventing the complex from forming aggregates, as shown in Figure 2. Upon adding more $\mathrm{H}$ homopolymer, a normal morphological change can be recognized, as shown by the morphological transition from a cylindrical structure (Figure $1 \mathrm{~g}$ ) to a lamellar one (Figure $1 \mathrm{~h}$ ) then to a reverse cylindrical structure (Figure 1i) whose cylinders are occupied by S. Further morphological transition can occur upon further addition of an $\mathrm{H}$ homopolymer up to an excess of 28-fold compared with the $\mathrm{P}$ component. This wide morphology change is remarkable if the transition at the top for
poly(styrene-b-2-vinylpyridine)/P blends is referenced. That is, the transition from a cylindrical structure to a lamellar one is certainly recognizable at a $\phi_{S}$ of 0.38 ; however, the added homopolymer starts to form a large independent domain because it segregates from an ordered domain structure, as shown in Figure 1d. Here, the solubility of the $\mathrm{H}$ homopolymer is much higher than that of the component homopolymer owing not only to the attracting force originating from hydrogen bonding but also to the induced neutral field for the $\mathrm{H}$ homopolymer formed by a $\mathrm{P} / \mathrm{H}$ complex. Thus, a very simple and easy method of controlling the morphology of a block copolymer is proposed in this study.

Another method of morphology control consists of utilizing the complex formation between electron acceptors on polymer chains and a metal salt. This section deals with the complexes formed between poly(4-vinylpyridine) and iron chloride as an example. ${ }^{45}$ The block copolymer sample was prepared by the reversible addition-fragmentation chain transfer polymerization method in bulk. The total molecular weight of the sample was $48 \mathrm{kDa}$, and the volume fraction of an $\mathrm{S}$ block was 0.77 . Hybrid sample films were obtained by casting a solvent from pyridine solutions. Figure 3 compares the structures of various mixtures. As an indicator of the blend ratio, the sample code $\mathrm{Fe}(X)$ was used, where $X$ denotes an iron/pyridine molar ratio. When $X$ is low, relatively unclear structures are observed; however, cylindrical structures are evident at $X$ equal to $0.5(2(\mathrm{e}))$ and $0.6(2(\mathrm{f}))$ even without staining, and the reason that it can happen without staining is that the blended salt increases the electron density. With the further addition of the salt, the cylindrical structure was transformed to a lamellar one when $\mathrm{Fe} / \mathrm{P}>0.7$.

The formation of periodic structures from block copolymer/metal salt hybrids suggests the possibility of very important promising materials such as optical- or electro-conductive materials because metal particles could also be dispersed periodically on a mesoscopic scale by utilizing this self-assembling manner. In addition, the basic idea can be applied widely to various organic/inorganic hybrid materials.

The formation of hierarchical structures from two block copolymer molecules that have multiple hydrogen bonding moieties

As demonstrated in the previous section, HBs between $\mathrm{H}$ and $\mathrm{P}$ can lead to several novel self-assembly processes if these two units are attached on chain ends of different polymers. Here, the combination of two block copolymers, poly(styrene-b-4-hydroxystyrene) (SH) $\left(M=86 \mathrm{kDa}, \phi_{S}=0.86\right)$ and poly(isoprene-b-2-vinylpyridine) (IP) $\left(M=80 \mathrm{kDa}, \phi_{I}=0.91\right)$, whose $\mathrm{H}$ and $\mathrm{P}$ sequences are fairly short, was first considered. ${ }^{46,47}$ Polymer samples were also synthesized by living anionic polymerizations in tetrahydrofuran, where an $\mathrm{SH}$ copolymer was prepared via poly(styrene-b-4-tert-butoxystyrene) followed by a hydrolysis reaction. Figure 4 compares the structures of the two blends, that is, poly(styrene-b-4-tert-butoxystyrene)/IP and SH/ IP. In Figure $4 a$, poly(styrene-b-4-tert-butoxystyrene) $/ \mathrm{IP}=50 / 50$ is a macrophase-separated structure of two component block copolymers without HBs, whereas in Figure 4b SH/IP appears to be an ordered lamellar nanophase-separated structure from two copolymers with HBs. One notices clearly the difference in the supramolecular selfassembly manner with and without HBs. Moreover, Figure 5a exhibits a detailed structure for the blend with HBs, which gives a hierarchical structure with periodically arranged isolated domains. The darkest domain probably indicates an $\mathrm{H} / \mathrm{P}$ mixed phase because the staining agent is $\mathrm{I}_{2}$. This domain is the cross-section of a complex lamellar structure with three phases. Clearly, two major components in the parent copolymers, that is, S in SH and I in IP, form alternating 

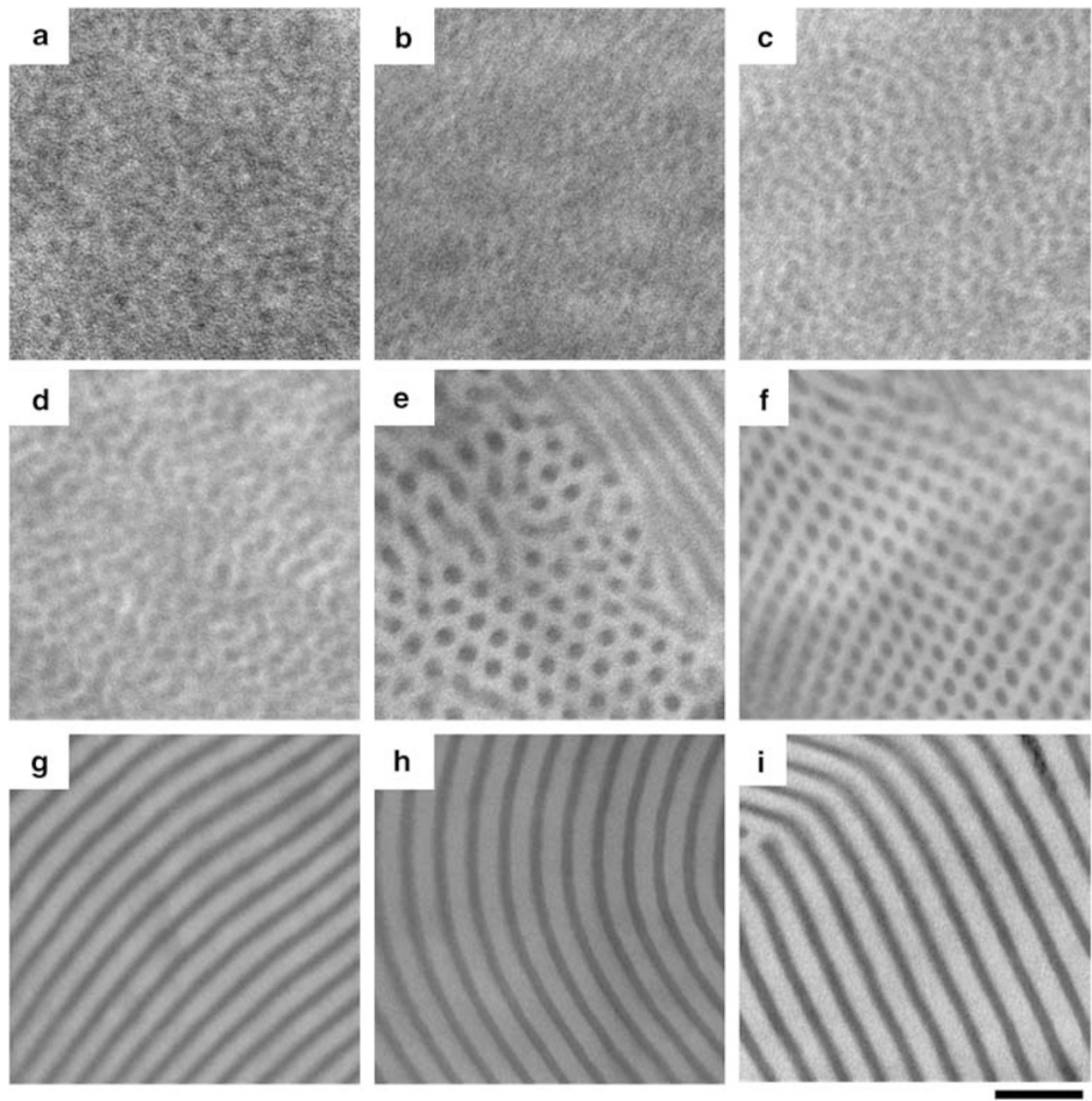

Figure 3 Transmission electron microscopy (TEM) images of $\mathrm{Fe}(X)(X=0.10 .9)$ : (a) $\mathrm{Fe}(0.1)$, (b) $\mathrm{Fe}(0.2),(\mathbf{c}) \mathrm{Fe}(0.3),(\mathbf{d}) \mathrm{Fe}(0.4),(\mathbf{e}) \mathrm{Fe}(0.5),(\mathbf{f}) \mathrm{Fe}(0.6)$, (g) $\mathrm{Fe}(0.7)$, (h) $\mathrm{Fe}(0.8)$ and (i) $\mathrm{Fe}(0.9)$. The scale bar represents $100 \mathrm{~nm}$. The samples were not stained with vapors but show strong contrast because of the electron density between the two phases that results from the incorporation of $\mathrm{FeCl}_{3}$ (which is indicated as the darker phase). Reprinted with permission from Noro et al. ${ }^{45}$ Copyright (C) 2010. American Chemical Society.

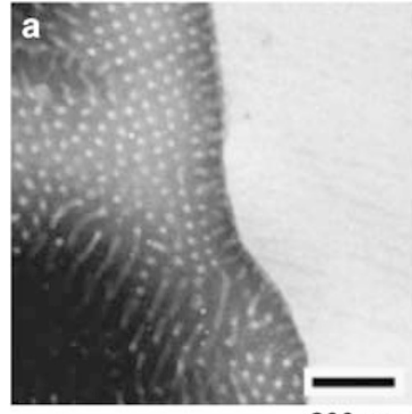

$200 \mathrm{~nm}$

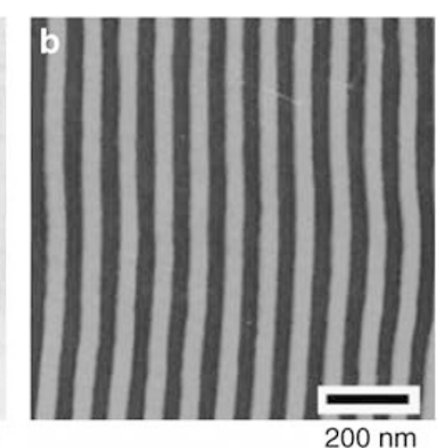

Figure 4 Transmission electron microscopy (TEM) images of an IP-91/ poly(styrene-4-tert-butoxystyrene)-91 $=50 / 50$ diblock copolymer blend film, showing a typical domain boundary between two microphase-separated structures (a), and TEM images of IP-91/SH-91 blends with weight ratios of 50/50 (b). Ultrathin sections were stained with osmium tetroxide. Reprinted with permission from Asari et al. ${ }^{47}$ Copyright (C) 2006. Nature Publishing Group.

lamellae, whereas an H/P mixed phase connects the two major phases. The alignment of the three domains is schematically shown in Figures $5 b$, and a possible arrangement of two molecules is drawn in Figure 5c, where the two molecules are self-assembled into star-like molecules whose arm number should be large. It should be noted that there are no junction points between the white $S$ phase and the gray I phase, which have principle roles in the lamellar structure.

The morphological change that depends on the blend ratios of $\mathrm{SH} /$ IP was studied in detail by using two extremely asymmetric copolymers, $\mathrm{SH}\left(M=71.3 \mathrm{kDa}, \phi_{S}=0.96\right)$ and IP $\left(M=57.6 \mathrm{kDa}, \phi_{I}=0.96\right){ }^{48}$ These two parent block copolymers show spherical structures (images are not presented here). When the complex formation is attained in blend solutions, the supramolecular self-assembling manner varies back again. Sample films were obtained by solvent casting from solutions of a tetrahydrofuran/toluene (1:1 by volume) mixed solvent. Toluene was added to control the polarity of a solvent so that the domain structure can form under moderate conditions. Figure 6 compares three typical images from the $\mathrm{IP} / \mathrm{SH}=50 / 50$ blend film stained with osmium tetroxide. Many dark loops in a bright matrix of SH can be seen in Figure 6a, whereas bright closed loops can be recognized in a dark matrix of IP in Figure $6 \mathrm{~b}$. These structures are part of the single-layered, vesicle-type structures through the formation of complexes from SH and IP molecules that are stoichiometrically associated to form block copolymer-type supramacromo- 


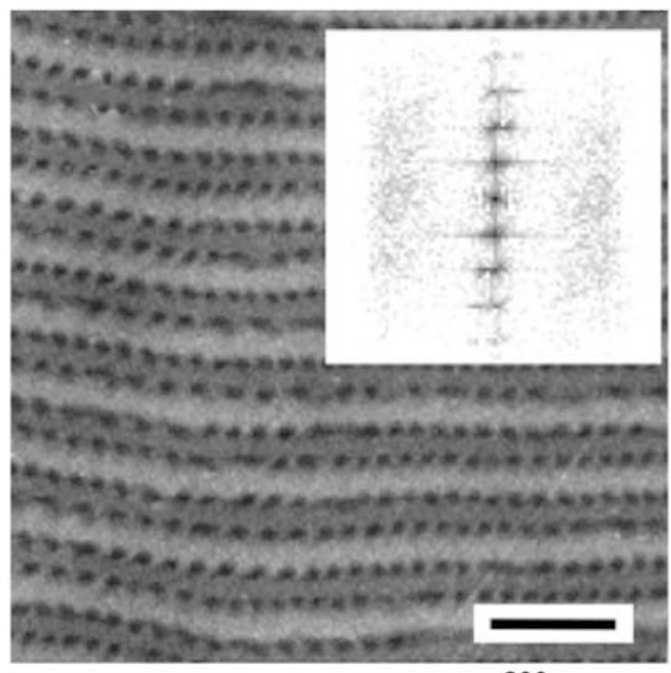

$200 \mathrm{~nm}$ b

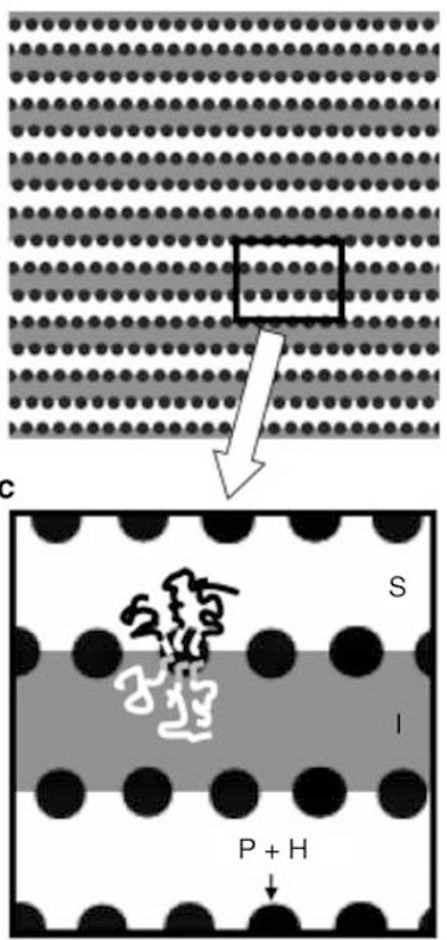

Figure 5 (a) An enlarged transmission electron microscopy (TEM) image of the IP-91/SH-91=50/50 blend. The inset shows the fast Fourier transform pattern for all of the areas in this TEM image. (b) A schematic drawing of a microdomain assembly for the corresponding blend observed in Figure 8a. (c) The enlarged domain assembly and a possible molecular arrangement within the structure of the complex. Reprinted with permission from Asari et al. ${ }^{46}$ Copyright (C) 2005. American Chemical Society.
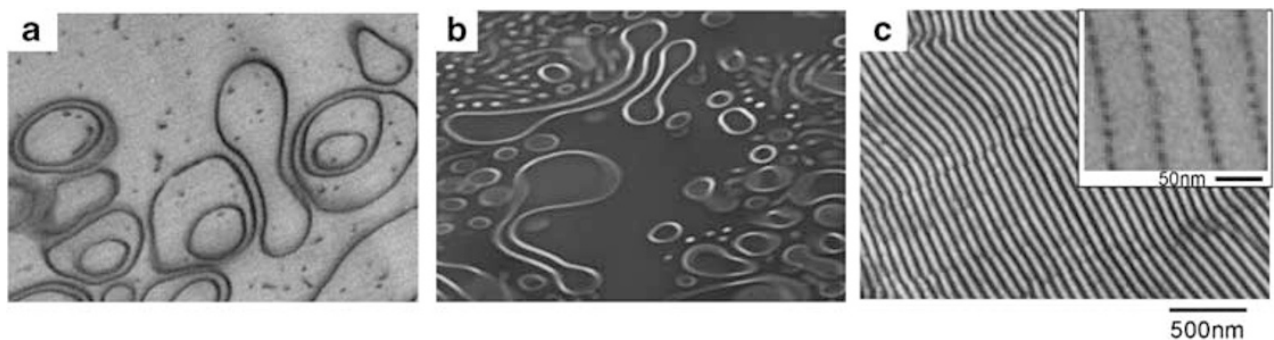

Figure 6 Transmission electron microscopy (TEM) images of an SH/IP=5/5 blend cast from tetrahydrofuran ((a) and (b)). (c) An image from a solution of a mixed solvent of tetrahydrofuran + toluene (1:1). Sample specimens were stained with osmium tetroxide. The inset in panel $\mathbf{c}$ shows a magnified image of $\mathbf{c}$ but stained with $\mathrm{I}_{2}$. Reprinted with permission from Dobrosielska et al. ${ }^{48}$ Copyright (C) 2010. American Chemical Society.

lecules. The $\mathrm{S}$ block in $\mathrm{SH}$ is embedded in $\mathrm{S}$ matrix as shown in Figure $6 \mathrm{a}$, whereas a polyisoprene (I) part is embedded in an I matrix as shown in Figure 6b. In contrast, when stoichiometric complex formation is realized in a one-to-one manner, a long-range lamellar structure can be created, as shown in Figure 6c. An enlarged figure in the inset of Figure $6 \mathrm{c}$ shows interesting isolated domains for an $\mathrm{H} / \mathrm{P}$ mixed phase, which have the role of giant junction points, and hence, the $S$ and I principle chains must be extended from this complex phase.

Figure 7, in turn, compares morphologies of the blends at various $\mathrm{SH} / \mathrm{IP}$ ratios from $1 / 9$ to $9 / 1$. It is easily recognized that a spherical structure at an SH/IP ratio of $1 / 9$ changes to a cylindrical structure at the ratios between $2 / 8$ and $3 / 7$ and then to a lamellar structure at the ratios between $4 / 6$ and $5 / 5$. Furthermore, morphologies continuously changes to a lamellar/cylindrical mixed structure at $6 / 4$, then to a reverse cylindrical structure at $7 / 3$ and finally to a spherical one at ratios between $8 / 2$ and $9 / 1$. This morphological transition was easily realized by merely blending at different ratios two molecules whose behavior is similar to that of block copolymers with different compositions.

Thus, we can provide a very simple idea of morphological transition to put it into practice just by changing the blend ratio of two parent molecules.

The combination of H/P complex formation and self-assembly of block copolymers can be further extended to the new field of polymer morphology. Figure 8 shows a structure of an IP/SH blend with a weight ratio of $1: 1$ whose parent molecules are both symmetric block copolymers, SH $\left(M_{\mathrm{n}}=74 \mathrm{kDa} \phi_{S}=0.49\right)$ and IP $\left(M_{\mathrm{n}}=84 \mathrm{kDa}\right.$ and $\left.\phi_{I}=0.54\right)$. Three phases, bright, dark and gray, can be clearly distin- 
a

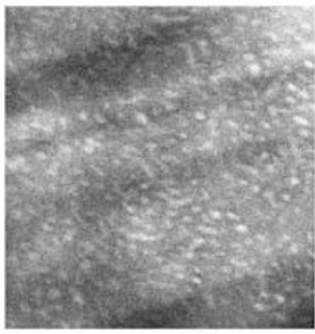

f

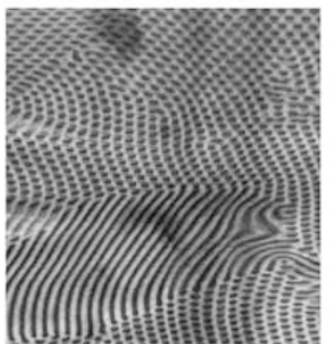

b

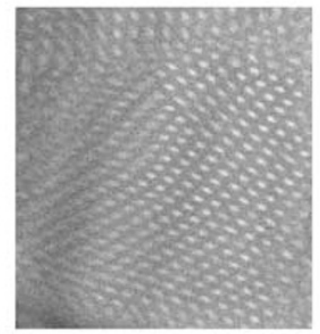

g

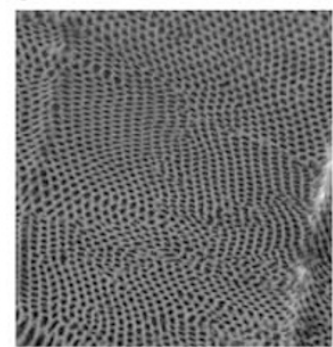

C

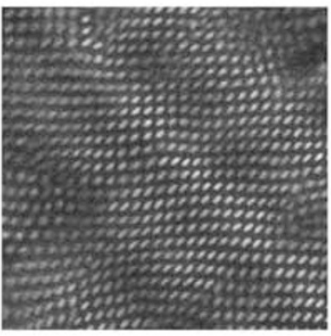

h

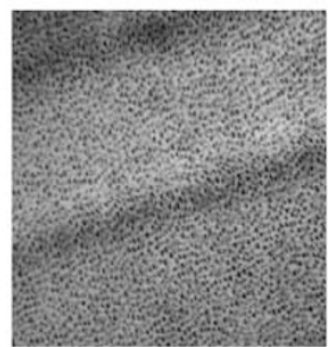

d

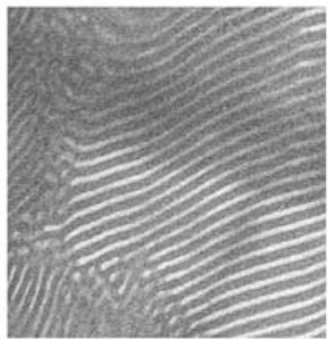

i

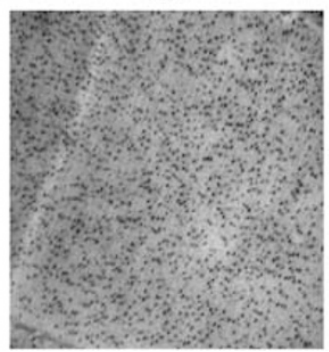

e $\quad 5: 5$

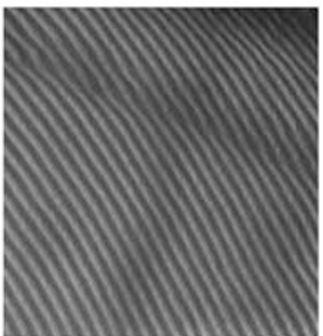

$500 \mathrm{~nm}$

Figure 7 Transmission electron microscopy (TEM) micrographs showing microphase-separated structures at different SH/IP molar ratios (staining agent osmium tetroxide) (a-i). Reprinted with permission from Dobrosielska et al. ${ }^{48}$ Copyright (C) 2010. American Chemical Society.

a

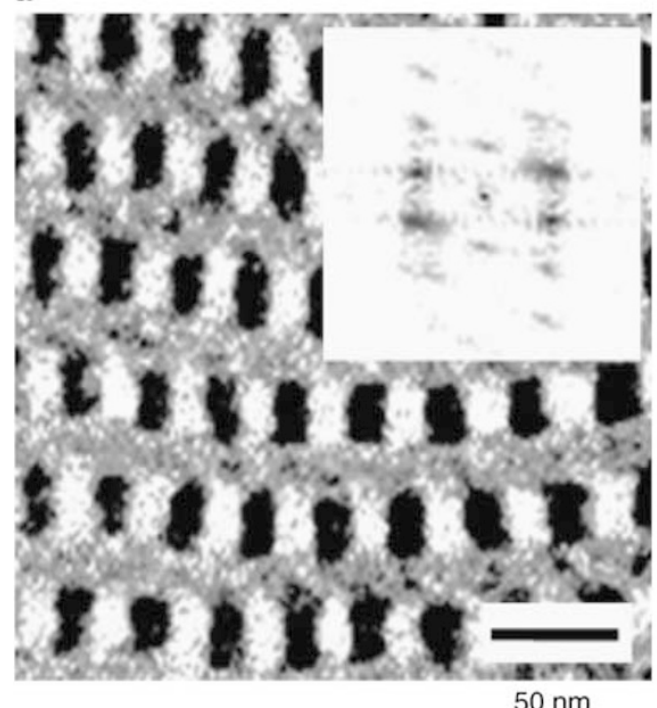

b

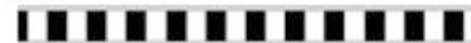

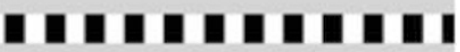

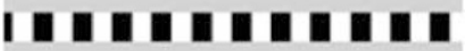

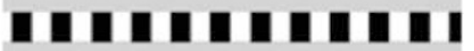

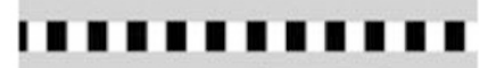

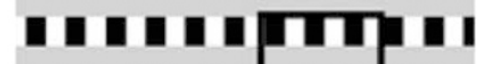

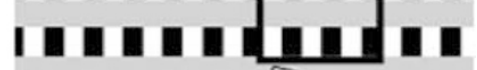

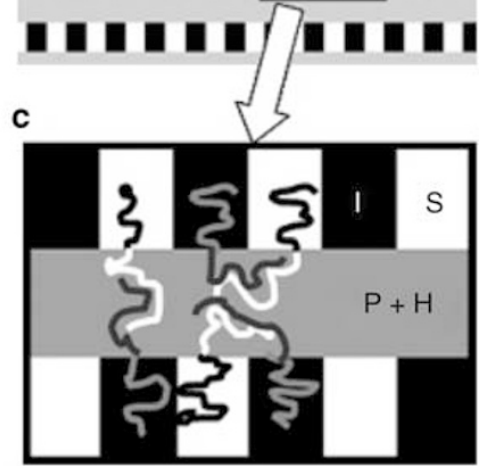

Figure 8 (a) An enlarged transmission electron microscopy (TEM) image of the IP-55/SH-55=1/1 blend. The inset shows the fast Fourier transform pattern for all of the areas in this TEM image. (b) A schematic drawing of the microdomain arrangement for the corresponding blend observed in Figure 5a. (c) The enlarged domain assembly and possible molecular arrangement within this structure. Reprinted with permission from Asari et al. ${ }^{46}$ Copyright $\left({ }^{C} 2005\right.$. American Chemical Society.

guished with a periodical arrangement; these phases correspond to S, I and an H/P mixed phase, respectively, because the sample specimen was stained with osmium tetroxide. This image shows the crosssection of a complex lamellar structure: the 'simple' lamella (with a gray color) consists of an H/P mixed phase with a volume fraction of $\sim 0.5$, and the other lamella is composed of two alternating isolated domains of S and I. This structure is one of the simplest hierarchical structures that possess two periodicities. In Figure $8 \mathrm{~b}$, the arrangement of three domains is drawn schematically, where bright and dark domains are alternating in both the vertical and horizontal directions. In Figure $8 \mathrm{c}$, the possible molecular conformations are also drawn schematically in the domains. It is somewhat surprising that there are 


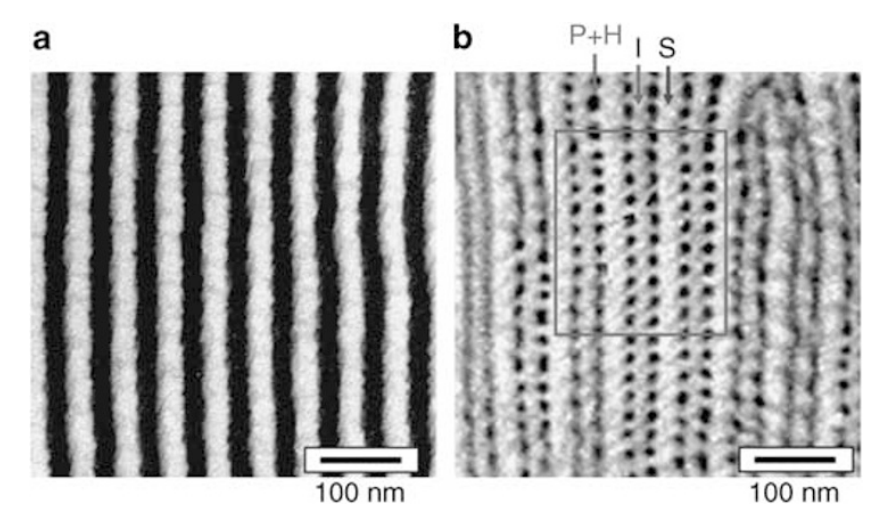

Figure 9 Transmission electron microscopy (TEM) images of the PIP-91/SH$91=1 / 1$ blend. Sample specimens were stained with (a) osmium tetroxide and (b) $I_{2}$. Reprinted with permission from Asari et al. ${ }^{49}$ Copyright (C) 2006. American Chemical Society. A full color version of this figure is available at Polymer Journal online.

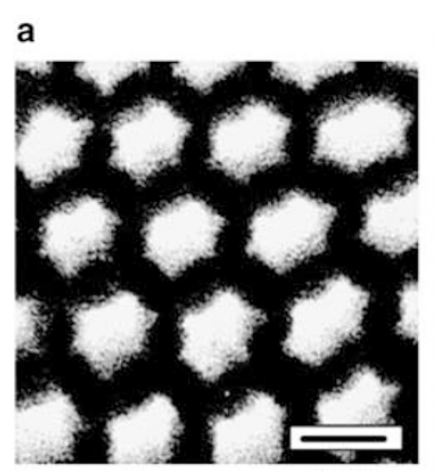

$50 \mathrm{~nm}$

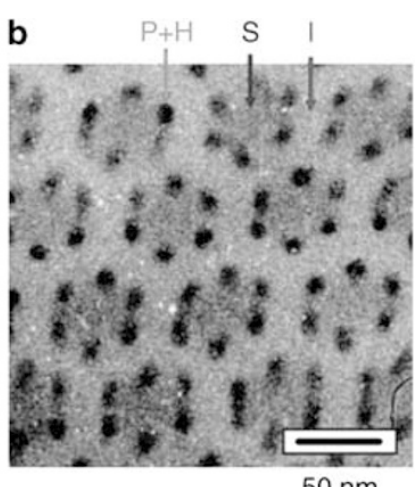

$50 \mathrm{~nm}$
Figure 10 Transmission electron microscopy (TEM) images of the PIP-91/ SH-91=2/1 blend. Sample specimens were stained with (a) osmium tetroxide and (b) $I_{2}$. Reprinted with permission from Asari et al. ${ }^{49}$ Copyright (C) 2006. American Chemical Society. A full color version of this figure is available at Polymer Journal online.

no junction points between the $S$ and I domains so that the S/I boundary tends to become a flat surface spontaneously.

Furthermore, the structure formation of diblock copolymer/triblock copolymer blends was investigated. Figure 9 compares structures of a mixture of an SH diblock copolymer $\left(M_{\mathrm{n}}=86 \mathrm{kDa}, \phi_{S}=0.86\right)$ and a PIP triblock copolymer $\left(M_{\mathrm{n}}=92 \mathrm{kDa}, \phi_{I}=0.93\right) .{ }^{49}$ Figure 9 a shows a blend of $\mathrm{SH} / \mathrm{PIP}=1 / 1$ with a simple alternating lamellar structure (osmium tetroxide staining), whereas the structure shown in Figure $9 \mathrm{~b}$ exhibits a hierarchical structure. This specimen was stained with $\mathrm{I}_{2}$ so that the $\mathrm{H} / \mathrm{P}$ mixed phase remained between the $\mathrm{S}$ and I lamellae, as shown in Figure 5 for SH/IP. Alternatively, Figure 10 represents another periodic structure, arising from an SH/PIP blend with a weight ratio of 2:1. In Figure 10a, the simple hexagonal bright domains, whose matrix should be an I phase, are aligned hexagonally, and these hexagonal domains correspond to an $\mathrm{S}$ phase because the specimen was stained with osmium tetroxide. In Figure 10b, the detailed structure is displayed, showing that isolated mixed domains are aligned at every corner of the hexagons; this alignment is another typical example of a hierarchical arrangement of domains, namely, this structure includes two periodicities: one is the hexagonal arrangement of the $\mathrm{S}$ domains, and the other is the occupancy of the mixed phase arranged hexagonally.
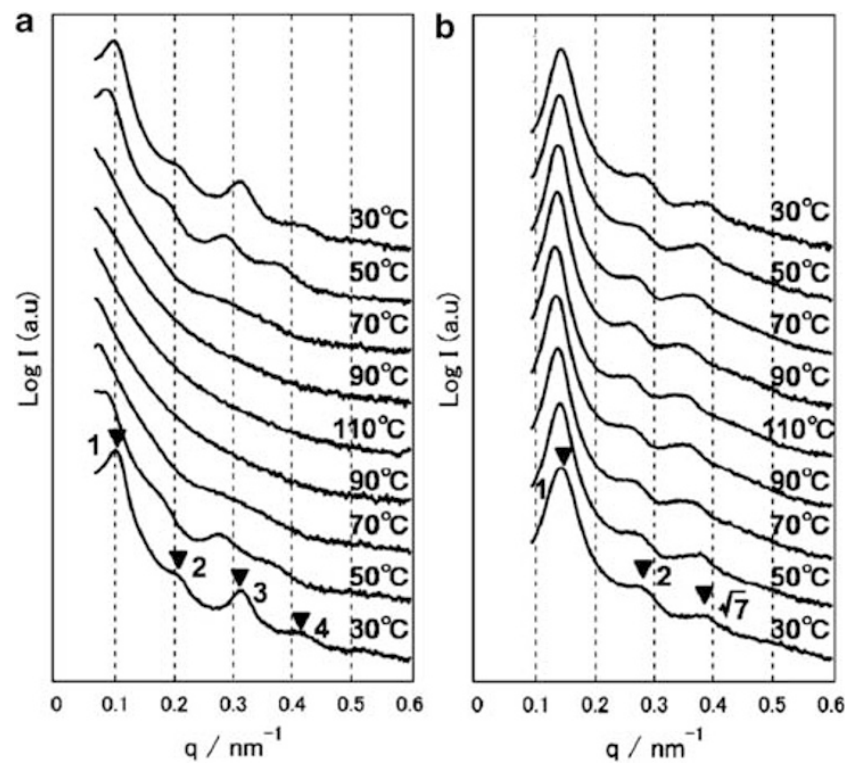

Figure 11 (a) Small-angle X-ray scattering profiles of block-type supramacromolecules (poly(3,4,5-trimethoxystyrene-PHS/PBA-P2VP) in an IL at various temperatures. The temperature increased from the bottom curve $\left(30^{\circ} \mathrm{C}\right)$ to the middle one $\left(110^{\circ} \mathrm{C}\right)$ and decreased from the middle to the top $\left(30^{\circ} \mathrm{C}\right)$. (b) Small-angle $\mathrm{X}$-ray scattering profiles of a block copolymer (poly(3,4,5-trimethoxystyrene-PBA) in an IL at various temperatures, which vary in the same manner as in (a). Reprinted with permission from Noro et al. ${ }^{50}$. Copyright ${ }^{C}$ 2009. American Chemical Society. a

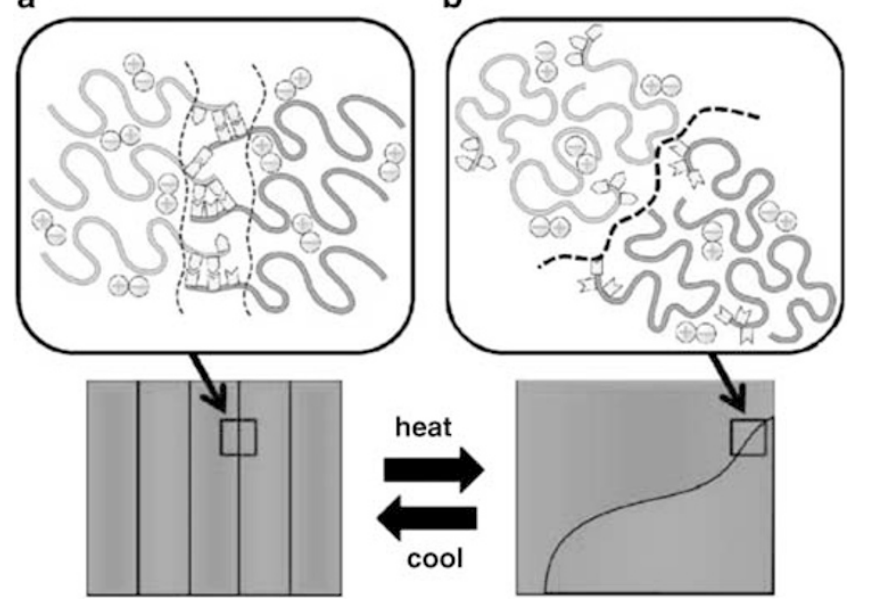

Figure $12 \mathrm{~A}$ schematic illustration of the thermoreversible morphology transition between nanophase separation and macrophase separation of block-type supramacromolecules with multiple hydrogen bonds at the nanoscopic length scale and molecular level: (a) at a low temperature; (b) at a high temperature. Complete macrophase separation is achieved at temperatures high enough to neglect the contribution of hydrogen bonding. Reprinted with permission from Noro et al. ${ }^{50}$ Copyright (C) 2009. American Chemical Society. A full color version of this figure is available at Polymer Journal online.

Thus, we are able to create a variety of hierarchical structures by combining the natural ability of supramolecular self-assembly of block copolymers and hydrogen bonding between an electron donor and an acceptor. This method is highly novel because it produces such high 
morphological variation; moreover, the method itself is very simple because only blending is required, so it can easily provide new morphological control strategies.

Another approach for dynamic morphology control was examined with two incompatible polymers bearing multiple hydrogen bonding moieties on each end. ${ }^{50}$ One of the polymers was poly(3,4,5-trimethoxystyrene)-b-PHS with a total molecular weight of $53 \mathrm{kDa}$, which has a degree of polymerization of 264 and 13 for poly(3,4,5-trimethoxystyrene) and PHS, respectively. The other polymer was poly(n-butyl acrylate)-b-P2VP (PBA-P2VP), which had a degree of polymerization of 412 and 15 for the PBA and P2VP sequences, respectively. Two samples were prepared by reversible addition-fragmentation chain transfer polymerization. Two polymers were blended in 1-ethyl-3-methylimidazolium bis(trifluoromethylsulfonyl)imide, which is known as an ionic liquid (IL), with the aid of tetrahydrofuran as a volatile solvent. The final fractions of poly(3,4,5-trimethoxystyrene)b-PHS, PBA-P2VP and IL were 20:20:60 for poly(3,4,5-trimethoxystyrene)-b-PHS: PBA-P2VP:IL, that is, a $40 \%$ concentrated polymer solution was prepared in an IL. A solution of a PTS-PBA block copolymer with a $40 \%$ polymer concentration was also prepared in the same IL as a reference sample. Small-angle X-ray scattering experiments were carried out by varying the temperature of the blend solution.

Figure 11a compares scattering curves for blend samples obtained at various temperatures. The intensity curve shown at the bottom of the figure was measured at $30^{\circ} \mathrm{C}$ and includes several integer numberordered peaks on the axis of the scattering vector, $\mathrm{q}$, indicating that the sample possesses a highly ordered, alternating lamellar structure in solution. The diffracted peaks gradually disappear as the temperature of the solution increases, and no peaks can be seen at $90^{\circ} \mathrm{C}$, indicating that the ordered, self-assembled structure is no longer present owing to the dissociation of the hydrogen bonded complex into component polymers at higher temperatures. However, in the upper part of Figure $11 \mathrm{a}$ it is apparent that diffracted peaks reappeared at lower temperatures owing to the re-formation of an ordered structure from two diblock copolymers as a result of re-association via hydrogen bonding with decreasing temperature. This thermoreversible phenomenon was confirmed by repeat experiments. In Figure 11b, a simple diblock copolymer system with a covalent bond shows no essential change in the scattering pattern with varying temperature, reflecting that the nanophase-separated structure of a block copolymer is naturally stable

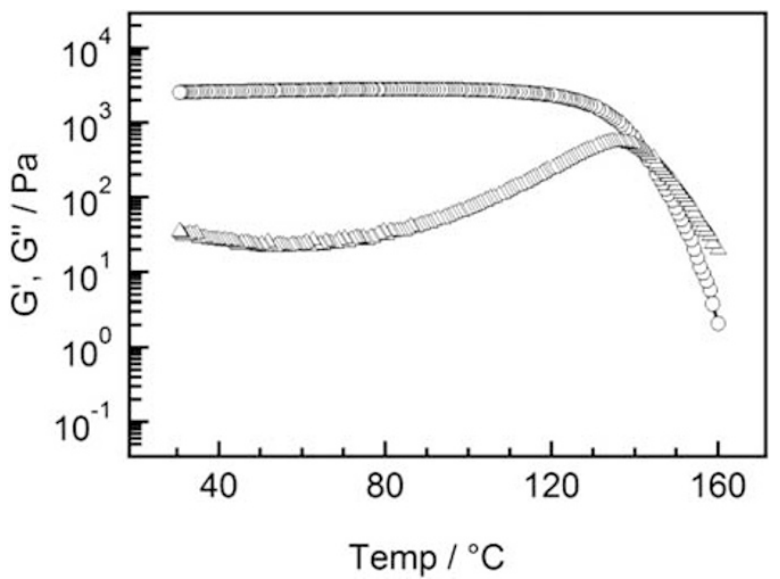

Figure 13 Storage $\left(G^{\prime}\right)$ and loss $\left(G^{\prime \prime}\right)$ moduli of supramolecular polymer gels in an ionic liquid (P2VP-poly(ethyl acrylate)-P2VP:PHS:EMITFSI=10:4:90 by weight) as a function of temperature. ${ }^{53}$ under the conditions used in the present study. In Figure 12, thermoreversible morphology transition that occurs as temperature increases is schematically displayed, showing thermoreversible dissociationassociation phenomena with and without HBs.

Thus, the reversible control of the nanoscopic-macroscopic structure was realized simply by changing the temperature. This behavior is directly related to the association-dissociation of hydrogen bonding between the two moieties connected at each end of the long chains. Therefore, this study suggests great potential of the applications to create temperature-switching or -tuning materials.

\section{Supramolecular gelation phenomena in telechelic-polymer/ multifunctional polymer systems}

In the previous section, we demonstrated that supramolecular assembly of a polymer block with multiple proton acceptor units (such as P2VP) and a polymer block with multiple proton donor units (such as PHS) provides novel multi-component supramacromolecular complexes. In these complexes, polymer blocks have hydrogenbonding moieties connected to form supramolecular giant junction points (or phases), representing a variety of hierarchical structures. The idea of connecting polymer blocks is very useful for practical materials, allowing the build-up not only of hierarchical nanophaseseparated structures of multi-component supramacromolecular complexes but also of supramacromolecular networks with novel viscoelastic properties. Although the presence of supramolecular polymer networks has been known empirically since the nineteenth century, for example, in gelatin (an aqueous solution of collagen), ${ }^{51}$ the concept of designing and building a supramolecular polymer network is relatively new. One of the studies at the early stage of supramolecular polymer networks was reported in the 1980s by Stadler et al. ${ }^{52}$ The general procedure for constructing supramolecular polymer networks proposed therein is the syntheses of telechelic polymers with many self-complementary, non-covalent bonded units on both ends. However, this process limits the study of a wide range of supramolecular polymer networks because special polymers must be synthesized. Here, we show a convenient preparation procedure in which polymers as components were synthesized via only a common reversible addition-fragmentation chain transfer polymerization method. In addition, we describe the interesting

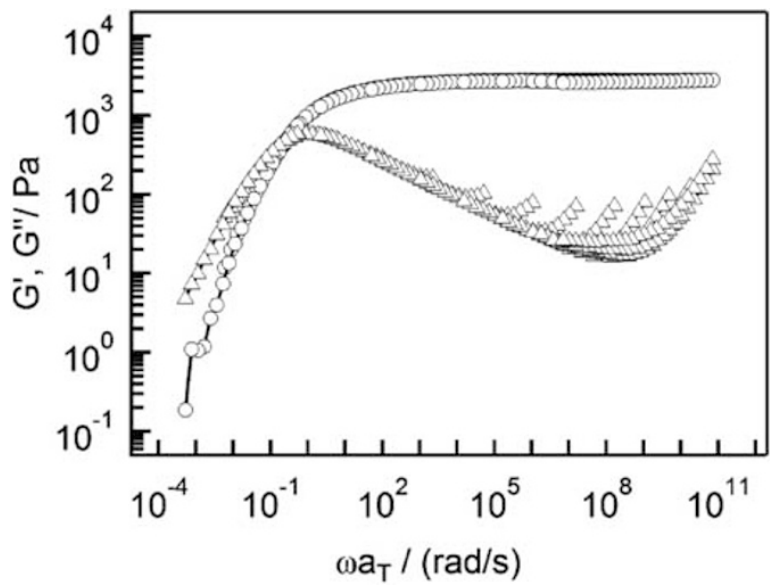

Figure 14 Master curves of storage $\left(G^{\prime}\right)$ and loss $\left(G^{\prime \prime}\right)$ moduli of supramolecular polymer gels in an ionic liquid (P2VP-poly(ethyl acrylate)P2VP:PHS:EMITFSI $=10: 4: 90$ by weight) as a function of reducedfrequency. The reference temperature was $140{ }^{\circ} \mathrm{C} .53$ 


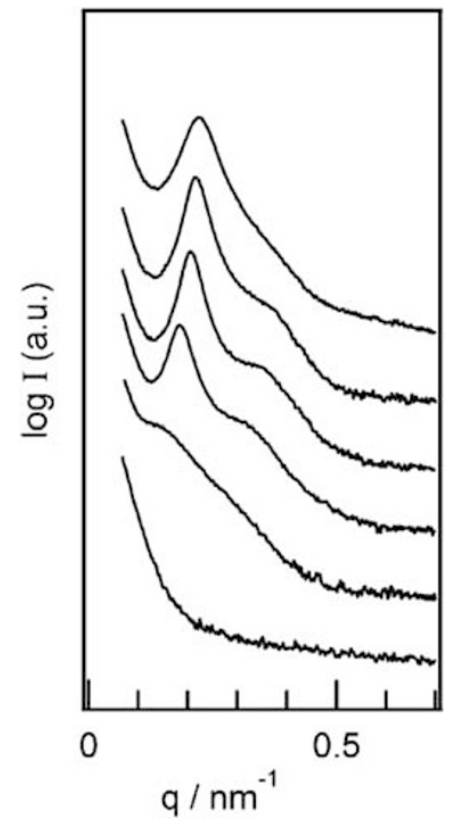

Figure 15 Small-angle X-ray scattering profiles of different blend samples at room temperature. The profiles are displayed in the order of $X$ (the fraction of PHS) from the bottom curve to the top: that is, the bottom curve is for $X=0$, the second from the bottom is for $X=0.5$, the third for $X=1.0$, the fourth for $X=2.0$, the fifth for $X=4.0$ and the top for $X=8.0 .{ }^{54}$

physical properties of supramolecular polymer networks, that is, supramolecular polymer gels (or supramacromolecular gels), at the macroscopic scale.

A useful design for building supramolecular networks of polymers is to blend two polymers in a non-volatile solvent. ${ }^{53,54}$ One of the two polymers used in this design is an $\mathrm{ABA}$ triblock copolymer that has two proton acceptor A blocks, and the other is a $\mathrm{C}$ homopolymer that functions as a multifunctional crosslinker with many proton-donor groups. A thermally stable IL is used as the non-volatile solvent and plasticizer to make the supramolecular polymer networks soft and flexible. The A blocks and a $\mathrm{C}$ homopolymer form a giant hydrogenbonded phase, whereas the B blocks form connecting hydrogenbonded phases, leading to the formation of supramolecular polymer networks (or supramacromolecular networks). As an example, P2VPb-poly(ethyl acrylate)-b-P2VP (P2VP-PEA-P2VP) and PHS were blended in a hydrophobic IL, 1-ethyl-3methylimidazolium bis(trifluoromethylsulfonyl) imide (EMITFSI), which dissolves PEA well. The blend formed a thermoreversible supramolecular polymer gel in an IL, which is called a supramacromolecular ion gel.

Figure 13 shows the storage $\left(\mathrm{G}^{\prime}\right)$ and loss $\left(\mathrm{G}^{\prime \prime}\right)$ moduli of gels with a weight ratio of P2VP-PEA-P2VP/PHS/EMITFSI of 10:4:90. The measurement condition was at a frequency of $0.3 \mathrm{rad} \mathrm{s}^{-1}$, a cooling rate of $1{ }^{\circ} \mathrm{C} \mathrm{min}^{-1}$ and a strain of $3 \%$. At high temperatures, $\mathrm{G}^{\prime \prime}$ is larger than $\mathrm{G}^{\prime}$, indicating a liquid behavior; however, with decreasing temperatures, $\mathrm{G}^{\prime}$ and $\mathrm{G}^{\prime \prime}$ achieved the same value at $141^{\circ} \mathrm{C}$, and $\mathrm{G}^{\prime}$ eventually became larger than $\mathrm{G}^{\prime \prime}$ at lower temperatures, suggesting a gel state with a plateau modulus of $\sim 3000$ Pa. This viscoelastic behavior was seen both on cooling and heating. Therefore, this gel was found to be thermoreversible. To understand the viscoelastic properties from the viewpoint of molecular dynamics, measurements of dynamic frequency sweeps at various temperatures were undertaken. Timetemperature superposition master curves are shown in Figure 14 with<smiles>CCOC(=O)C(CC(C)(C)C(=O)O)SC(=S)SC(CC(C)(C)C(=O)O)C(=O)OCC</smiles>
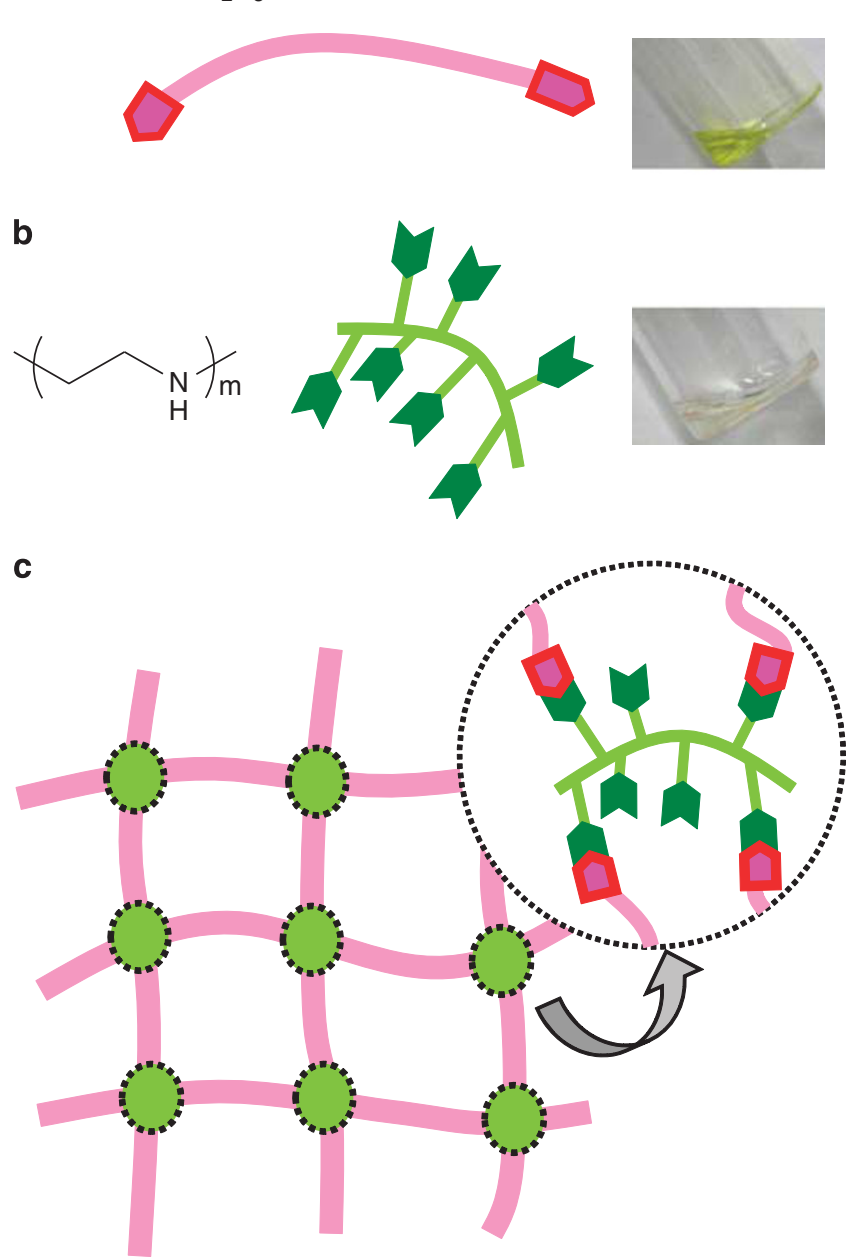

Figure 16 Schematic illustrations of building blocks ((a) and (b)) and a supramolecular polymer network via hydrogen bonding (c). Reprinted with permission from Noro et al. ${ }^{55}$ Copyright (C) 2011. RSC Publishing.

a reference temperature of $140{ }^{\circ} \mathrm{C}$. The curves superposed comparatively well, except that systematic deviations occurred at higher reduced-frequencies. A liquid behavior was observed at low reduced frequencies, whereas a remarkably wide plateau with at least 11 orders of magnitude of $\omega$ was seen at higher frequencies. The crossover of $\mathrm{G}^{\prime}$ and $\mathrm{G}^{\prime \prime}$ was $0.24 \mathrm{rad} \mathrm{s}^{-1}$, which indicates an estimated longest relaxation time of $4.2 \mathrm{~s}$ at $140^{\circ} \mathrm{C}$. These observations suggest that the longest relaxation time of the gel increases up to at least $10^{11} \mathrm{~s}$ at room temperature because of the formation of HBs between a given P2VP block and the PHS homopolymer.

This distinct supramolecular gelation is also related to the nanophase separation between a hydrogen-bonded phase and an IL-soluble phase. Figure 15 shows the small-angle X-ray scattering profiles of the blends of P2VP-PEA-P2VP/PHS/EMITFSI, where the weight ratio of the blends was set as 10:X:90 $(X=0,0.5,1,2,4,8)$. There is no distinct 
scattering peak in the profile of the 10:0:90 blend, shown at the bottom, indicating that there is no particular nanostructure. As the amount of PHS added increases, some scattering peaks are detected, although the 10:0.5:90 blend with a small amount of PHS shows quite a broad peak. All of these results suggest that the addition of PHS induces nanophase separation in blends, as well as supramolecular gelation.

By simplifying the above molecular design, supramolecular polymer gels can be prepared easily even in a bulk state. ${ }^{55}$ Figure 16 displays one of the simplest models of a supramolecular polymer network together with its building blocks. One building block used as a bridging strand is carboxy-terminated telechelic poly(ethyl acrylate), and the other building block used as a multifunctional crosslinker is polyethyleneimine with many amino groups. Both polymers are liquids. The carboxylic acid and the amine moieties form acid-base complexes with HBs, which build up supramolecular networks of liquid polymers, in other words, supramolecular polymer gels in bulk. Because the supramolecular polymer gels are 'bulk', the gels possess a higher modulus of approximately $1 \mathrm{MPa}$, making them much more robust under conditions of stress than supramolecular polymer gels in solvents such as ILs (as shown by $10 \mathrm{wt} \%$ gels in an IL having a plateau modulus of $3000 \mathrm{~Pa}$; Figures 13 and 14). The crossover of $\mathrm{G}^{\prime}$ and $\mathrm{G}^{\prime \prime}$, which could be regarded as a gel-liquid transition temperature, is also much lower than that of the previously constructed gels with multiple HBs at crosslinks in an IL, probably because there are fewer HBs at a crosslink or a junction point in bulk supramolecular polymer gels than in the previously constructed gels in an IL.

As stated above, studies of the molecular design and physical properties of supramolecular gels are just beginning; 56 therefore, these gels will need to be explored further in the future for the development of attractive materials, such as advanced rubber materials that self-heal via hydrogen bonding, ${ }^{57,58}$ or ionic hydrogen bonding. ${ }^{59}$

\section{CONCLUSION}

The combination of the self-assembling ability of block copolymers together with non-covalent bonding interactions produces many interesting structures with a mesoscopic length scale. As mentioned above, non-covalent bonding interactions include hydrogen bonding, ionic interactions and metal-to-ligand coordination. The materials created by applying this new idea of supramacromolecular selfassembly could lead to a variety of attractive high-performance materials, for optical-, electromagnetic- and stimuli-responsive purposes.

\section{CONFLICT OF INTEREST}

The authors declare no conflict of interest.

\section{ACKNOWLEDGEMENTS}

This work was partially supported through the Global COE Program in Chemistry entitled 'Elucidation and Design of Materials and Molecular Functions' at Nagoya University and a Grant-in-Aid for Scientific Research on the Priority Area 'Soft Matter Physics' (No. 18068008 for Y.M.) by the Ministry of Education, Culture, Sports and Science, and Technology of Japan. This work was also partially supported by the Japan Society for the Promotion of Science through a Grant-in-Aid for Scientific Research (A) (No. 22245038 for Y.M.) and a Grant-in-Aid for challenging Exploratory Research (No. 23655213 for A.N.).
1 Lehn, J.- M. Supramolecular Chemistry: Concepts and Perspectives 1-271 (Wiley-VCH, Weinheim, 1995).

2 Russell, T. P., Jerome, R., Charlier, P. \& Foucart, M. The microstructure of block copolymers formed via ionic interactions. Macromolecules 21, 1709-1717 (1988).

3 Haraguchi, M., Inomata, K. \& Nose, T. Miscibility of associated polymer blends in good solvent.2. One-end-aminated polystyrene/one-end-sulfonated poly(ethylene glycol) blends in toluene. Polymer 37, 3611-3614 (1996).

4 Inomata, K., Haraguchi, M. \& Nose, T. Morphology of associated polymer blends: oneend-aminated polystyrene one-end-carboxylated or sulfonated poly(ethylene glycol). Polymer 37, 4223-4228 (1996).

5 Huh, J., Park, H. J., Kim, K. H., Park, C. \& Jo, W. H. Giant thermal tunability of the lamellar spacing in block-copolymer-like supramolecules formed from binary-endfunctionalized polymer blends. Adv. Mater. 18, 624 (2006).

6 Pispas, S., Floudas, G., Pakula, T., Lieser, G., Sakellariou, S. \& Hadjichristidis, N. Miktoarm block copolymer formation via ionic interactions. Macromolecules 36, 759-763 (2003).

7 Noro, A., Tamura, A., Wakao, S., Takano, A. \& Matsushita, Y. Stoichiometric effects on nanostructures of block- and graft-type supramacromolecules via acid-base complexation. Macromolecules 41, 9277-9283 (2008).

8 Qian, J. \& Wu, F. P. Preparation of supramolecular graft copolymers and the subsequent formation of pH-sensitive vesicles. Chem. Mater. 21, 758-762 (2009).

9 Sijbesma, R. P., Beijer, F. H., Brunsveld, L., Folmer, B. J. B., Hirschberg, J.H.K.K., Lange, R. F. M., Lowe, J. K. L. \& Meijer, E. W. Reversible polymers formed from self-complementary monomers using quadruple hydrogen bonding. Science 278, 1601-1604 (1997).

10 Berl, V., Schmutz, M., Krische, M. J., Khoury, R. G. \& Lehn, J. M. Supramolecular polymers generated from heterocomplementary monomers linked through multiple hydrogen-bonding arrays-formation, characterization, and properties. Chemistry $\mathbf{8}$, 1227-1244 (2002)

11 Yang, X. W., Hua, F. J., Yamato, K., Ruckenstein, E., Gong, B., Kim, W. \& Ryu, C. Y. [Supramolecular AB diblock copolymers]. Angewandte Chemie 43, 6471-6474 (2004).

12 Binder, W. H., Bernstorff, S., Kluger, C., Petraru, L. \& Kunz, M. J. Tunable materials from hydrogen-bonded pseudo block copolymers. Adv. Mater. 17, 2824 (2005).

13 Noro, A., Nagata, Y., Takano, A. \& Matsushita, Y. Diblock-type supramacromolecule via biocomplementary hydrogen bonding. Biomacromolecules 7, 1696-1699 (2006).

14 Feldman, K. E. et al. Polymers with multiple hydrogen-bonded end groups and their blends. Macromolecules 41, 4694-4700 (2008).

15 Yamauchi, K., Lizotte, J. R. \& Long, T. E. Thermoreversible poly(alkyl acrylates) consisting of self-complementary multiple hydrogen bonding. Macromolecules 36, 1083-1088 (2003).

16 Yamauchi, K., Kanomata, A., Inoue, T. \& Long, T. E. Thermoreversible polyesters consisting of multiple hydrogen bonding (MHB). Macromolecules 37, 3519-3522 (2004).

17 Ruokolainen, J., ten Brinke, G., Ikkala, O., Torkkeli, M. \& Serimaa, R. Mesomorphic structures in flexible polymer surfactant systems due to hydrogen bonding: poly (4-vinylpyridine)-pentadecylphenol. Macromolecules 29, 3409-3415 (1996).

18 Ruokolainen, J., Tanner, J., Ikkala, O., ten Brinke, G. \& Thomas, E. L. Direct imaging of self-organized comb copolymer-like systems obtained by hydrogen bonding: poly (4-vinylpyridine)-4-nonadecylphenol. Macromolecules 31, 3532-3536 (1998).

19 Ruokolainen, J., Makinen, R., Torkkeli, M., Makela, T., Serimaa, R., ten Brinke, G. \& Ikkala, O. Switching supramolecular polymeric materials with multiple length scales. Science 280, 557-560 (1998).

20 Ikkala, O. \& ten Brinke, G. Functional materials based on self-assembly of polymeric supramolecules. Science 295, 2407-2409 (2002).

21 Dai, J., Goh, S. H., Lee, S. Y. \& Siow, K. S. Interpolymer complexation between poly (p-vinylphenol) and pyridine-containing polymers. Polym. J. 26, 905-911 (1994)

22 Pan, J., Chen, M. F., Warner, W., He, M. Q., Dalton, L. \& Hogen-Esch, T. E. Synthesis and self-assembly of diblock copolymers through hydrogen bonding semiquantitative determination of binding constants. Macromolecules 33, 7835-7841 (2000).

23 Jiang, S. M., Gopfert, A. \& Abetz, V. Novel morphologies of block copolymer blends via hydrogen bonding. Macromolecules 36, 6171-6177 (2003).

24 Beck, J. B. \& : Rowan, S. J. Multistimuli, multiresponsive metallo-supramolecular polymers. J. Am. Chem. Soc. 125, 13922-13923 (2003).

25 Weng, W. G., Beck, J. B., Jamieson, A. M. \& Rowan, S. J. Understanding the mechanism of gelation and stimuli-responsive nature of a class of metallo-supramolecular gels. J. Am. Chem. Soc. 128, 11663-11672 (2006).

26 Chan, Y. N. C., Schrock, R. R. \& Cohen, R. E. Synthesis of single silver nanoclusters within spherical microdomains in block copolymer films. J. Am. Chem. Soc. 114, 7295-7296 (1992).

27 Boal, A. K., Ilhan, F., DeRouchey, J. E., Thurn-Albrecht, T., Russell, T. P. \& Rotello, V. $M$. Self-assembly of nanoparticles into structured spherical and network aggregates. Nature 404, 746-748 (2000).

28 Ho, R. M., Lin, T., Jhong, M. R., Chung, T. M., Ko, B. T. \& Chen, Y. C. Phase transformation in self-assembly of the gold/poly(4-vinylpyridine)-b-poly(e-caprolactone) hybrid system. Macromolecules 38, 8607-8610 (2005).

29 Lin, T., Ho, R. M. \& Ho, J. C. Phase behavior in self-assembly of inorganic/poly(4vinylpyridine)-b-poly(epsilon-caprolactone) hybrid. Macromolecules 42, 742-751 (2009).

30 Lin, T., Li, C. L., Ho, R. M. \& Ho, J. C. Association strength of metal ions with poly(4vinylpyridine) in horganic/poly(4-vinylpyridine)-b-poly(epsilon-caprolactone) hybrids. Macromolecules 43, 3383-3391 (2010). 
31 Tsutsumi, K., Funaki, Y., Hirokawa, Y. \& Hashimoto, T. Selective incorporation of palladium nanoparticles into microphase-separated domains of poly(2-vinylpyridine)block-polyisoprene. Langmuir 15, 5200-5203 (1999).

32 Adachi, M., Okumura, A., Sivaniah, E. \& Hashimoto, T. Incorporation of metal nanoparticles into a double gyroid network texture. Macromolecules 39, 7352-7357 (2006).

33 Lee, D. H., Kim, H. Y., Kim, J. K., Huh, J. \& Ryu, D. Y. Swelling and shrinkage of lamellar domain of conformationally restricted block copolymers by metal chloride. Macromolecules 39, 2027-2030 (2006).

34 Lee, D. H., Han, S. H., Joo, W., Kim, J. K. \& Huh, J. Phase behavior of poly styreneblock-poly (4-vinylpyridine) copolymers coordinated by metal chloride. Macromolecules 41, 2577-2583 (2008).

35 Mendoza, C., Gindy, N., Gutmann, J. S., Fromsdorf, A., Forster, S. \& Fahmi, A. Block copolymers with gold nanoparticles: correlation between structural characteristics and mechanical properties. Macromolecules 42, 1203-1211 (2009).

36 Mendoza, C., Pietsch, T., Gutmann, J. S., Jehnichen, D., Gindy, N. \& Fahmi, A. In situ synthesis and alignment of Au nanoparticles within hexagonally packed cylindrical domains of diblock copolymers in bulk. Langmuir 25, 9571-9578 (2009).

37 Dobrosielska, K., Wakao, S., Takano, A. \& Matsushita, Y. Nanophase-separated structures of $\mathrm{AB}$ block copolymer/C homopolymer blends with complementary hydrogen-bonding interactions. Macromolecules 41, 7695-7698 (2008).

38 Dobrosielska, K., Wakao, S., Suzuki, J., Noda, K., Takano, A. \& Matsushita, Y. Effect of homopolymer molecular weight on nanophase-separated structures of AB block copolymer/C homopolymer blends with hydrogen-bonding interactions. Macromolecules 42, 7098-7102 (2009).

39 Matsushita, Y., Shimizu, K., Nakao, Y., Choshi, H., Noda, I. \& Nagasawa, M. Preparation and characterization of poly(2-vinylpyridine)s with narrow molecular-weight distributions. Polym. J. 18, 361-366 (1986).

40 Matsushita, Y., Nakao, Y., Saguchi, R., Choshi, H. \& Nagasawa, M. Studies of styrene and 2-vinylpyridine block copolymers-preparation and characterization. Polym. J. 18, 493-499 (1986).

41 Hirai, A., Yamaguchi, K., Takenaka, K., Suzuki, K., Nakahama, S. \& Yamazaki, N. [Polymerization of monomers containgn functional-groups protected by trialkylsilyl groups 1 . Synthesis of poly(4-vinylphenol) by means of anionic living polymerization]. Makromol. Chem. 3, 941-946 (1982).

42 Hirahara, K., Takano, A., Yamamoto, M., Kazama, T., Isono, Y., Fujimoto, T. \& Watanabe, O. Fabrication of solid polymer electrolyte based on block-graft copolymer. 1.Precision synthesis and characterization of polystyrene-block-[poly(p-hydroxystyrene)-graft-poly (ethylene oxide)]-block-polystyrene. React. Funct. Polym. 37, 169-182 (1998).

43 Moskala, E. J., Howe, S. E., Painter, P. C. \& Coleman, M. M. On the role of intermolecular hydrogen-bonding in miscible polymer blends. Macromolecules 17, 1671-1678 (1984).

44 Pehlert, G. J., Painter, P. C. \& Coleman, M. M. Functional group accessibility in hydrogen-bonded polymer blends 3. Steric shielding effects. Macromolecules 31 , 8423-8424 (1998).
45 Noro, A., Sageshima, Y., Arai, S. \& Matsushita, Y. Preparation and morphology control of block copolymer/metal salt hybrids via solvent-casting by using a solvent with coordination ability. Macromolecules 43, 5358-5364 (2010).

46 Asari, T., Matsuo, S., Takano, A. \& Matsushita, Y. Three-phase hierarchical structures from $A B / C D$ diblock copolymer blends with complemental hydrogen bonding interaction. Macromolecules 38, 8811-8815 (2005).

47 Asari, T., Matsuo, S., Takano, A. \& Matsushita, Y. Preparation and characterization of diblock copolymers of the AB and CD types and their self-assembled structure by hydrogen bonding interaction. Polym. J. 38, 258-263 (2006).

48 Dobrosielska, K., Takano, A. \& Matsushita, Y. Creation of hierarchical nanophaseseparated structures via supramacromolecular self-assembly from two asymmetric block copolymers with short interacting sequences giving hydrogen bonding interaction. Macromolecules 43, 1101-1107 (2010).

49 Asari, T., Arai, S., Takano, A. \& Matsushita, Y. Archimedean tiling structures from ABA/ $\mathrm{CD}$ block copolymer blends having intermolecular association with hydrogen bonding. Macromolecules 39, 2232-2237 (2006).

50 Noro, A., Yamagishi, H. \& Matsushita, Y. Thermoreversible morphology transition from block-type supramacromolecules via hydrogen bonding in an ionic liquid. Macromolecules 42, 6335-6338 (2009).

51 Harrington, W. F. \& Vonhippel, P. H. The structure of collagen and gelatin. Adv. Protein Chem. 16, 1-138 (1961).

52 Muller, M., Dardin, A., Seidel, U., Balsamo, V., Ivan, B., Spiess, H. W. \& Stadler, R. Junction dynamics in telechelic hydrogen bonded polyisobutylene networks. Macromolecules 29, 2577-2583 (1996).

53 Noro, A., Matsushita, Y. \& Lodge, T. P. Thermoreversible supramacromolecular ion gels via hydrogen bonding. Macromolecules 41, 5839-5844 (2008).

54 Noro, A., Matsushita, Y. \& Lodge, T. P. Gelation mechanism of thermoreversible supramacromolecular ion gels via hydrogen bonding. Macromolecules 42, 58025810 (2009).

55 Noro, A., Hayashi, M., Ohshika, A. \& Matsushita, Y. Simple preparation of supramolecular polymer gels via hydrogen bonding by blending two liquid polymers. Soft Matter 7, 1667-1670 (2010).

56 Feldman, K. E., Kade, M. J., Meijer, E. W., Hawker, C. J. \& Kramer, E. J. Model transient networks from strongly hydrogen-bonded polymers. Macromolecules 42 , 9072-9081 (2009).

57 Cordier, P., Tournilhac, F., Soulie-Ziakovic, C. \& Leibler, L. Self-healing and thermoreversible rubber from supramolecular assembly. Nature 451, 977-980 (2008).

58 Montarnal, D., Tournilhac, F., Hidalgo, M., Couturier, J. L. \& Leibler, L. Versatile one-pot synthesis of supramolecular plastics and self-healing rubbers. J. Am. Chem. Soc. 131, 7966 (2009).

59 Noro, A., Ishihara, K. \& Matsushita, Y. Nanophase-separated supramolecular assemblies of two functionalized polymers via acid-base complexation. Macromolecules 44, 6241-6244 (2011). 